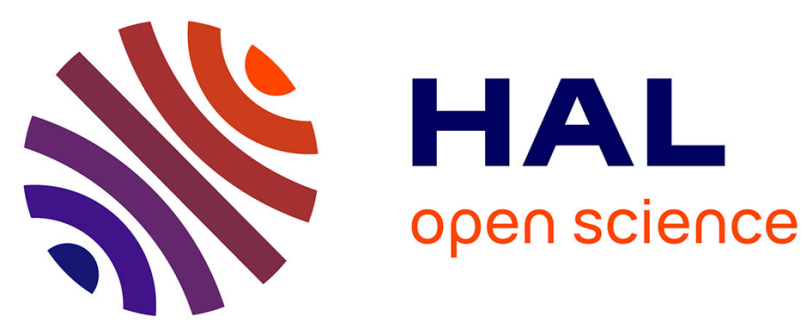

\title{
Interpretation of Cluster data on chorus emissions using the backward wave oscillator model
}

\author{
V. Yu. Trakhtengerts, A. G. Demekhov, E.E. Titova, B. V. Kozelov, O. \\ Santolik, D S Gurnett, Michel Parrot
}

\section{- To cite this version:}

V. Yu. Trakhtengerts, A. G. Demekhov, E.E. Titova, B. V. Kozelov, O. Santolik, et al.. Interpretation of Cluster data on chorus emissions using the backward wave oscillator model. Physics of Plasmas, 2004, 11 (4), pp.1345-1351. 10.1063/1.1667495 . insu-02928459

HAL Id: insu-02928459

https://hal-insu.archives-ouvertes.fr/insu-02928459

Submitted on 2 Sep 2020

HAL is a multi-disciplinary open access archive for the deposit and dissemination of scientific research documents, whether they are published or not. The documents may come from teaching and research institutions in France or abroad, or from public or private research centers.
L'archive ouverte pluridisciplinaire HAL, est destinée au dépôt et à la diffusion de documents scientifiques de niveau recherche, publiés ou non, émanant des établissements d'enseignement et de recherche français ou étrangers, des laboratoires publics ou privés. 


\section{Interpretation of Cluster data on chorus emissions using the backward wave oscillator model}

Cite as: Physics of Plasmas 11, 1345 (2004); https://doi.org/10.1063/1.1667495

Submitted: 25 August 2003 . Accepted: 24 November 2003 . Published Online: 24 March 2004

V. Y. Trakhtengerts, A. G. Demekhov, E. E. Titova, B. V. Kozelov, O. Santolik, D. Gurnett, and M. Parrot

\section{ARTICLES YOU MAY BE INTERESTED IN}

Electron holes in phase space: What they are and why they matter

Physics of Plasmas 24, 055601 (2017); https://doi.org/10.1063/1.4976854

Electron-acoustic solitary waves in the Earth's inner magnetosphere

Physics of Plasmas 25, 022905 (2018); https://doi.org/10.1063/1.5007907

Parameter spaces for linear and nonlinear whistler-mode waves

Physics of Plasmas 20, 072110 (2013); https://doi.org/10.1063/1.4816022

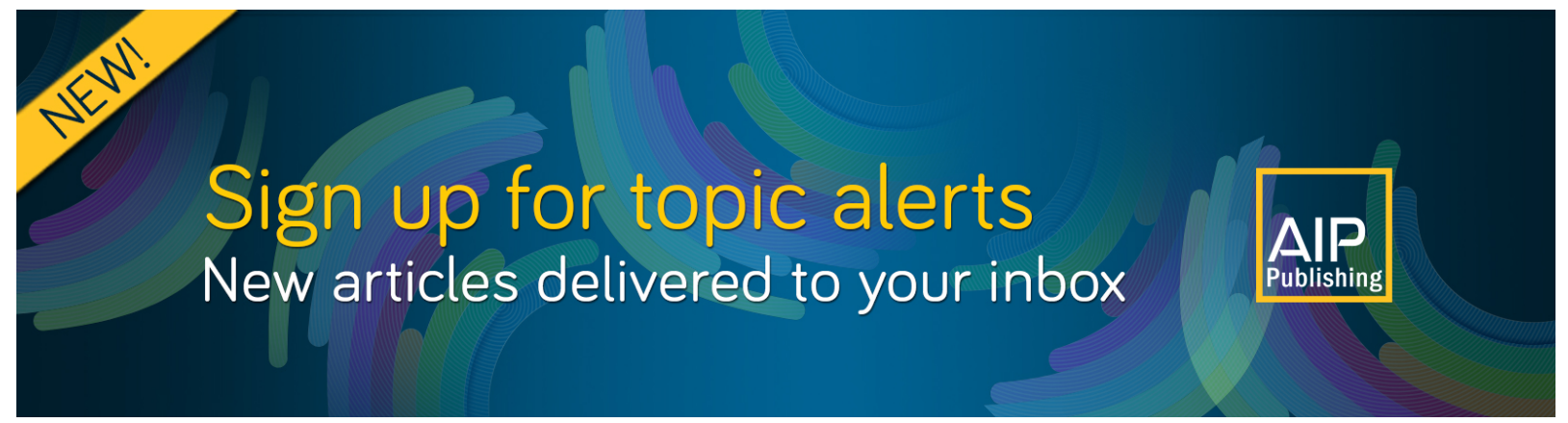




\title{
Interpretation of Cluster data on chorus emissions using the backward wave oscillator model
}

\author{
V. Y. Trakhtengerts and A. G. Demekhov \\ Institute of Applied Physics, Nizhny Novgorod, Russia \\ E. E. Titova and B. V. Kozelov \\ Polar Geophysical Institute, Apatity, Russia \\ O. Santolik \\ Charles University, Prague, Czech Republic \\ D. Gurnett \\ University of Iowa, Iowa City, Iowa \\ M. Parrot \\ CNRS/LPCE, Orleans, France
}

(Received 25 August 2003; accepted 24 November 2003; published online 24 March 2004)

\begin{abstract}
The measurements of chorus emissions by four closely separated Cluster spacecraft provide important information concerning the chorus generation mechanism. They confirm such properties of the wave source as their strong localization near the equatorial cross section of a magnetic flux tube, an almost parallel average wave-vector direction with respect to the geomagnetic field, and an energy flux direction pointing outward from the generation region. Inside this region, Cluster discovered strong temporal and spatial variations in the amplitude with correlation scale lengths of the order of $100 \mathrm{~km}$ across the magnetic flux. The wave electric field reached $30 \mathrm{mV} / \mathrm{m}$, and the maximum growth and damping rates are of the order of a few hundreds of $\mathrm{s}^{-1}$. These and other properties of the detected chorus emissions are discussed here in relation with the backward wave oscillator mechanism. According to this mechanism, a succession of whistler wave packets is generated in a small near-equatorial region with temporal and spatial characteristics close to the Cluster data. Amplitudes and frequency spectra, as well as dynamical features of the Poynting flux of chorus are estimated and compared with the Cluster measurements. (C) 2004 American Institute of Physics. [DOI: 10.1063/1.1667495]
\end{abstract}

\section{INTRODUCTION}

Chorus is the most wide-spread type of natural discrete electromagnetic ELF/VLF emissions, which accompany the main and recovery phases of magnetic storms. ${ }^{1,2}$ Apparently, it is the most important type of electromagnetic emssions in the magnetosphere, providing precipitation of bulk energetic electrons from radiation belts ${ }^{3,4}$ and essentially contributing to the acceleration of $\mathrm{MeV}$ electrons. ${ }^{5}$

At the same time, chorus emissions remain the most intriguing signals among natural ELF/VLF radiation. They consist of a succession of discrete elements with rising frequency, with a repetition period of $T \sim 0.1-1 \mathrm{~s}$. According to ground-based observations the typical duration of a chorus event is $0.5-1 \mathrm{~h}$ or more. Satellite measurements ${ }^{6,7}$ show that chorus is generated in a narrow region near the equatorial cross section of a magnetic flux tube, and appears as whistler waves whose wave vector $\vec{k}$ is close to the direction of the magnetic field $\vec{H}$. As a rule, chorus is accompanied by hiss emissions which serve as a lower frequency background for the discrete elements. For more detailed information about chorus emissions one can refer to Refs. 1, 2, and 8 .

It is generally accepted that the generation mechanism of chorus is connected with the cyclotron instability (CI) of radiation belt $(\mathrm{RB})$ electrons. The similarity of the spectral forms of chorus elements and of triggered ELF/VLF emissions has stimulated the application of the theory of triggered signals to explain chorus generation. Helliwell ${ }^{9}$ suggested a phenomenological model of the generation of discrete emissions, based on the second-order cyclotron resonance. This model stimulated further analytical ${ }^{10}$ and computational ${ }^{11}$ studies (see Ref. 12 for more detail). New step in the quantitative theory of chorus generation was made in Ref. 13, where the backward wave oscillator (BWO) generation mechanism was suggested.

Backward-wave oscillators are well known in electronics, ${ }^{14}$ but application of this knowledge to the magnetospheric conditions is not trivial. The matter of fact is that laboratory BWOs use beams of electron oscillators (electrons gyrating in the magnetic field) with small dispersion in both perpendicular $\left(v_{\perp}\right)$ and parallel $\left(v_{\|}\right)$velocities with respect to the geomagnetic field: $\Delta v_{\perp} / v_{\perp} \ll 1$ and $\Delta v_{\|} / v_{\|} \ll 1$. Under RB conditions, such beams are absent. However, it was shown in Ref. 13 that not only delta-like distribution function but also sufficiently sharp gradients (step-like deformations) in the velocity space can lead to the BWO generation regime. According to Ref. 15, such step-like features appear naturally upon the development of the cyclotron instability 
(CI) of RB and generation of noise-like ELF emissions.

Actually, if there is an upper-frequency cutoff $\omega_{\max }$ in the spectrum of noiselike emissions, then it follows from the cyclotron resonance condition

$$
\omega-\omega_{H}=k_{\|} v_{\|}
$$

that the resonant particles occupy the region bounded from below in the equatorial values of the field-aligned velocity:

$$
\left|v_{\| L}\right| \geqslant\left|v_{\|}\right|=\left|\frac{\omega_{H}-\omega}{k_{\|}}\right| \geqslant\left|\frac{\omega_{H L}-\omega_{\max }}{k_{\| L}}\right| .
$$

Here, $\omega_{H}$ is the electron gyrofrequency, and the subscript " $L$ " denotes the values in the equatorial plane. In the resonant region, the distribution function is isotropized due to the velocity diffusion, while this process is absent in the nonresonant region. Therefore, a step is formed at the boundary $v_{\| L}=\left(\omega_{\max }-\omega_{H L}\right) / k_{\| L}$. This is shown in Fig. 1 on the example of numerical calculation. ${ }^{15}$ Upper panel shows the whistler wave energy density $\varepsilon(\xi)$ and gain $\Gamma(\xi)$, where $\xi$ $=1-\omega_{0} / \omega, \omega_{0}=\omega_{H L} / \beta_{*}$, and $\beta_{*}=\left(\omega_{p L} / \omega_{H L}\right)^{2}(v / c)^{2}$. Lower panel shows the pitch-angle distribution and pitchangle diffusion coefficient $D\left(x=\sin \vartheta_{L}\right.$, where $\vartheta_{L}$ is the equatorial pitch angle). The snapshots are taken for subsequent time moments during generation of a noise-like emission in the simulation. One can see that a moving sharp step is formed in this case, accompanied by excitation of higherfrequency noise-like emissions.

\section{BASIC FEATURES OF THE BWO GENERATION MODEL OF CHORUS EMISSIONS}

The BWO model of chorus generation is based on a specific modulation of the distribution function of energetic electrons in the course of CI development, which leads to the appearance of a coherent energetic electron clusters and generation of discrete ELF/VLF electromagnetic emission. This interaction manifests itself as the absolute cyclotron instability which develops in the local region near the equatorial cross section of the magnetic flux tube. The length $l_{\mathrm{BwO}}$ of the generation region along the magnetic field line is equal to $^{9,12,13,16}$

$$
l_{\mathrm{BWO}} \simeq 1.76\left(\lambda R_{E}^{2} L^{2} / 6\right)^{1 / 3},
$$

where $\lambda$ is the wavelength, $R_{E}$ is the Earth's radius, and $L$ is the Mcllwain parameter. The factor 1.76 was obtained in numerical study of the linear BWO theory in the parabolic magnetic field. ${ }^{16}$ At the initial stage of chorus generation, such a specific evolution is seen as a step-like deformation function of the energetic electrons in field-aligned velocities (Fig. 1). In the case of a step-like distribution function, the absolute instability is realized, if the wave growth rate $\gamma_{\text {step }}$ for the step-like distribution function (Fig. 1) of energetic electrons exceeds some threshold value $\gamma_{\mathrm{thr}}$. The growth rate of the absolute (BWO) instability is equal to ${ }^{13}$

$$
\gamma_{\mathrm{BWO}}=\frac{\pi^{2}}{8 T_{0}} Q(Q-1) \text {. }
$$

Here,
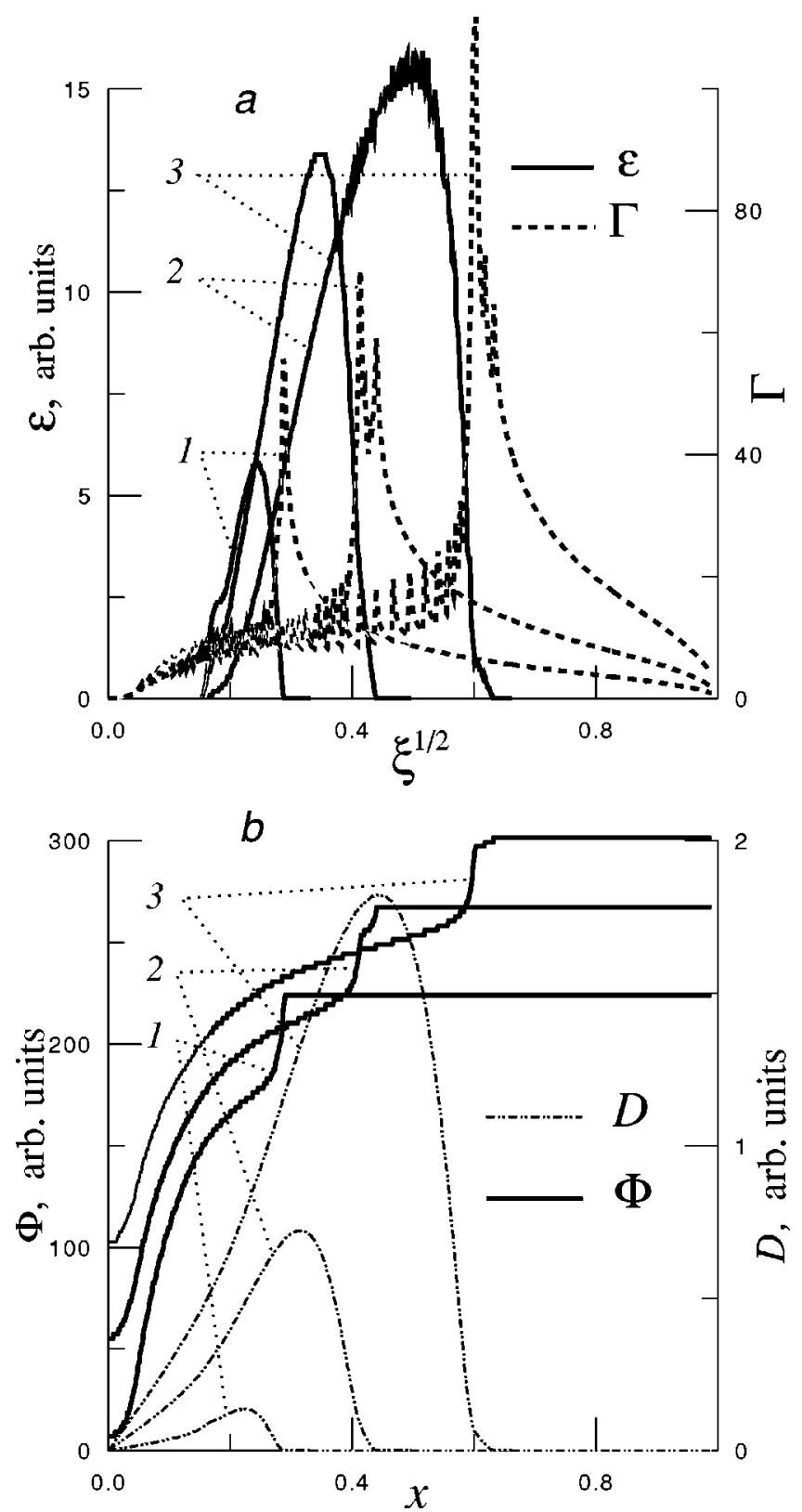

FIG. 1. Formation of sharp drops (steps) in the velocity space (from Ref. $15)$.

$$
Q=\frac{\gamma_{\text {step }}}{\gamma_{\text {thr }}}=\frac{2 l_{\text {BWO }}}{\pi\left(v_{g} v_{\text {step }}\right)^{1 / 2}} \gamma_{\text {step }}
$$

is the dimensionless parameter showing the excess of the threshold, which is proportional to the hydrodynamic growth rate $\gamma_{\text {step }}=0.25 \omega_{p h} \beta_{\perp} b^{1 / 2}$,

$$
\gamma_{\mathrm{thr}}=\pi / T_{0}, \quad T_{0}=l_{\mathrm{BwO}}\left(\frac{1}{v_{g}}+\frac{1}{v_{\text {step }}}\right),
$$

$\omega_{p h}=\left(4 \pi e^{2} N_{h} / m\right)^{1 / 2}, N_{h}$ is the density of energetic electrons, $\beta_{\perp}=u_{\perp 0} / c, u_{\perp 0}$ is the characteristic velocity across the magnetic field, $b=\Delta N_{h} / N_{h}$ is the relative height of the step, which is typically about $10 \%-20 \%$ in our simulations, and $v_{g}$ and $v_{\text {step }}$ are the group velocity and velocity at the step, respectively. 


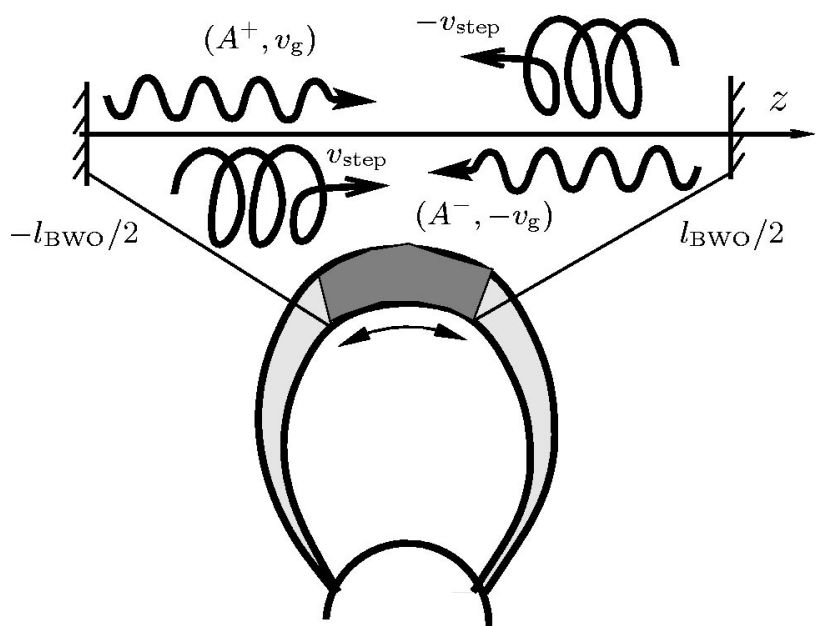

FIG. 2. Schematic picture of magnetospheric BWO.

If the inequality $\gamma_{\text {step }}>\gamma_{\text {thr }}$ is satisfied, then the amplitude of a whistler wave which is in cyclotron resonance with the step, grows exponentially in time in the local region near the equatorial cross section with the growth rate given in (4). This absolute instability is due to the phase bunching of step electrons, which produces a positive feedback between a whistler-mode wave and a hot-plasma mode supported by the energetic electron "beam."

Actually, if the step is formed, two "beams" of energetic electrons appear with a sharp front in the field-aligned velocity component, which move in $\pm z$ directions (Fig. 2) with the velocities $\pm v_{\text {step }}$ and generate chorus independently in opposite directions. If the growth rate $\gamma_{\mathrm{BWO}}$ is sufficiently large, the nonlinear phase bunching effects result in the transition from the growing phase of the wave amplitude to the damping phase. This corresponds to some bifurcations in the generation regimes, with transition from the stationary to the periodic one, and then to a stochastic regime. ${ }^{14}$ Chorus generation can be associated with the two latter regimes.

Dynamics of these regimes is described by the wave equation with nonlinear current of energetic electrons (see, e.g., Ref. 10).

There is a rich experience in computer modeling of cyclotron whistler-mode generation, based on this equation (e.g., Refs. 11, 17, and 18). However, a computer simulation of the BWO regime with the step-like distribution is rather complicated and has not been realized yet. The situation is simplified in the case of a model with wave-particle interaction in two discrete points located symmetrically to the equator, $z_{1,2}= \pm l_{\mathrm{BWO}} / 2$, where the cyclotron resonance is fulfilled (Fig. 2). Theoretical and experimental studies of the laboratory BWOs with the initial electron distribution as $\delta$-function in the velocity space $\left(F \propto \delta\left(v_{\perp}-v_{\perp 0}\right) \delta\left(v_{\|}\right.\right.$ $\left.-v_{\| 0}\right)$ ) showed that the two-point interaction model preserves the basic qualitative and many quantitative features of the BWO generation, which are observed in distributed BWO systems. ${ }^{14}$ Similar model can be obtained for the steplike distribution function. Leaving the details for a separate paper, we write the final nonlinear equation for the whistler wave amplitude in the following form (for chorus propagating in the $+z$ direction):

$$
\begin{aligned}
A^{+}\left(l_{\mathrm{BWO}} / 2, t\right)= & q A^{+}\left(l_{\mathrm{BWO}} / 2, t-T_{0}\right) \\
& \times\left[1-\left(A^{+}\left(l_{\mathrm{BWO}} / 2, t-T_{0}\right)\right)^{2}\right],
\end{aligned}
$$

where $A^{+}\left(l_{\mathrm{BWO}} / 2, t\right)$ is the dimensionless chorus amplitude at the point $z=l_{\mathrm{BWO}} / 2$, and the parameter $q$ is related to the $\mathrm{BWO}$ growth rate as

$$
q=1+2 \gamma_{\mathrm{BWO}} T_{0}=1+\frac{\pi^{2}}{8} Q(Q-1)
$$

[see Eqs. (4)-(6) for notations]

For the opposite direction,

$$
\begin{aligned}
A^{-}\left(-l_{\mathrm{BWO}} / 2, t^{\prime}\right)= & q A^{-}\left(-l_{\mathrm{BWO}} / 2, t^{\prime}-T_{0}\right) \\
& \times\left[1-\left(A^{-}\left(-l_{\mathrm{BWO}} / 2, t^{\prime}-T_{0}\right)\right)^{2}\right] .
\end{aligned}
$$

As $q$ increases, Eqs. (7) and (9) demonstrate the transition between all the above-mentioned generation regimes, i.e., from the stationary generation with constant amplitude at 1 $<q<2$, to a regime with periodic amplitude modulation at $q>2$, and to the stochastic generation at $q>2.3$.

The amplitude $\left|H_{\sim}^{ \pm}\right|$of the wave magnetic field is related with $A^{ \pm}$as follows:

$$
\left|H_{\sim}^{ \pm}\right|=\frac{2^{3 / 2} \omega_{p h} m v_{\text {step }}^{2}}{k u_{\perp 0} e l_{\mathrm{BWO}}} b^{1 / 2} A^{ \pm},
$$

where $k$ is the wave vector $\left(\vec{k} \| \vec{H}_{0}\right)$, and the other notations have been introduced above.

It is clear from Eqs. (7) and (9) that the saturation amplitude is

$$
A^{ \pm} \sim 1
$$

Using the definitions (4) to (6) and (10), we can obtain from (11) the maximum value of $\left|H_{\sim}\right|$ for $q \simeq 2$,

$$
\left|H_{\sim}\right|_{\mathrm{m}} \simeq \frac{\delta m}{e k \beta_{\perp}} \gamma_{\mathrm{BWO}}^{2},
$$

where $\delta \simeq 6$ for $\omega / \omega_{H L} \simeq 0.5, \quad \beta_{\perp}=u_{\perp 0} / c$, and $\gamma_{\mathrm{BWO}}$ $\simeq \pi^{2} /\left(4 T_{0}\right)$ [see (4)].

It is also possible to estimate the characteristic chorus amplitude in a different way. In the case of the absolute instability, such an estimate can be obtained from the relation $^{12}$

$$
\Omega_{\mathrm{tr}} / \gamma_{\mathrm{BWO}} \simeq 32 /(3 \pi),
$$

where $\gamma_{\mathrm{BWO}}$ is determined by (4) and the trapping frequency $\Omega_{\text {tr }}$ is equal to

$$
\Omega_{\mathrm{tr}}=\left(k u_{\perp 0} \omega_{H L}\left|H_{\sim}\right| / H_{L}\right)^{1 / 2} .
$$

From (13) and (14) we have

$$
\left|H_{\sim}\right|=\frac{10 m}{e k \beta_{\perp}} \gamma_{\mathrm{BWO}}^{2} .
$$

As one could expect, the estimates (12) and (15) are close to each other. 
TABLE I. Summary of chorus parameters in the BWO model. Numerical estimates are obtained for the event shown in Fig. 3 and studied experimentally in Ref. 20. Basic parameters are $L=4.4$, cold plasma density $N_{c} \sim 2 \mathrm{~cm}^{-3}$, and $\omega / \omega_{H L} \approx 0.45$ [see Fig. 3(a)]. From that, we obtain the resonant parallel energy $W_{\text {res }}=(m / 2)\left(\left(\omega_{H L}-\omega\right) / k\right)^{2} \simeq 62 \mathrm{keV}$ and the wavelength $\lambda \sim 26 \mathrm{~km}$. The flux density of energetic electrons is assumed to be $S \sim 4 \times 10^{8} \mathrm{~cm}^{-2} \mathrm{~s}^{-1}$.

\begin{tabular}{|c|c|c|c|c|c|}
\hline & $\begin{array}{l}\text { Length of } \\
\text { generation } \\
\text { region, } l_{\text {BWo }}\end{array}$ & $\begin{array}{c}\text { Characteristic } \\
\text { period of } \\
\text { succession, } \\
T>T_{0}\end{array}$ & $\begin{array}{c}\text { Growth rate } \\
\gamma_{\mathrm{BWO}}\end{array}$ & $\begin{array}{l}\text { Frequency drift } \\
\qquad \mathrm{d} f / \mathrm{d} t\end{array}$ & $\begin{array}{l}\text { Wave amplitude } \\
\qquad\left|H_{\sim}\right|\end{array}$ \\
\hline \multirow[b]{2}{*}{ Theory } & $1.76\left(\frac{R_{E}^{2} L^{2}}{k}\right)^{1 / 3}$ & $>l_{\mathrm{BWO}}\left(\frac{1}{v_{g}}+\frac{1}{v_{\text {step }}}\right)$ & $\frac{\pi^{2}}{8 T_{0}} Q(Q-1)$ & $0.5 \gamma_{\mathrm{BWO}}^{2}$ & $\frac{10 m c}{e k u_{\perp 0}} \gamma_{\mathrm{BWO}}^{2}$ \\
\hline & $2600 \mathrm{~km}$ & $>0.025 \mathrm{~s}$ & $\begin{array}{l}\sim 1.2 \times 10^{2} \mathrm{~s}^{-1} \\
\left(b=\frac{\Delta N_{h}}{N_{h}}=0.17\right)\end{array}$ & $0.7 \times 10^{4} \mathrm{~Hz} \mathrm{~s}^{-1}$ & $\sim 100 \mathrm{pT}$ \\
\hline Observations & $\approx 2000 \mathrm{~km}$ & $\begin{array}{c}0.02-0.5 \mathrm{~s} \\
\text { [see Fig. } 4(\mathrm{a})]\end{array}$ & $34-420 \mathrm{~s}^{-1}$ & $1.5 \times 10^{4} \mathrm{~Hz} \mathrm{~s}^{-1}$ & $100-300 \mathrm{pT}$ \\
\hline
\end{tabular}

Equations (7) and (9) describe the dynamics of the wave amplitude at the base frequency, corresponding to the step velocity, and determine retriggering of the next element at this frequency. Formation of the entire chorus element is related to the sideband instability, ${ }^{19}$ which results in the generation of new frequencies. ${ }^{17}$ Rigorous consideration of the chorus element formation requires sophisticated computer simulations of the complete problem, which are beyond the scope of this paper. We restrict ourselves to the qualitative consideration, suggesting that a chorus element is formed as a succession of sidebands separated from each other by the frequency interval $\Delta f \sim \Omega_{\mathrm{tr}} /(2 \pi)$ and growing with the temporal rate $\gamma_{\mathrm{BWO}}$. Taking into account (13), we obtain

$$
\frac{\mathrm{d} f}{\mathrm{~d} t} \sim \Delta f \gamma_{\mathrm{BWO}} \sim 0.5 \gamma_{\mathrm{BWO}}^{2}
$$

This estimation is close to the one which follows from the optimum condition for cyclotron interaction of an electron with a whistler wave in an inhomogeneous magnetic field. ${ }^{10,12}$

In the following sections, we use the obtained relations for the discussion of specific chorus data from Cluster.

\section{PROPERTIES OF VLF DYNAMICS IN THE MAGNETOSPHERIC BWO}

Relations (3) to (4), (12), and (16) allow one to determine the basic parameters of chorus emission. These parameters are given in Table I, which summarizes their analytical expressions, their estimates for the particular values of the magnetic field, the cold plasma density and the radiation electron parameters, and their comparison with Cluster data

(a)

(b)

$$
\text { UT: }
$$$$
\text { MLat (deg): } \quad-5.73
$$

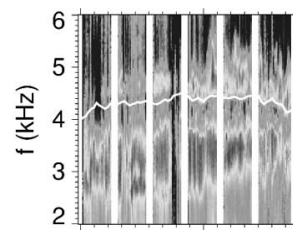

$$
\begin{array}{ll}
0 & 5 \\
- & 0
\end{array}
$$

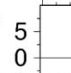$$
\text { UT: } 0839
$$$$
\mathrm{R}\left(\mathrm{R}_{\mathrm{E}}\right): \quad 4.41
$$

MLT (h): 21.08
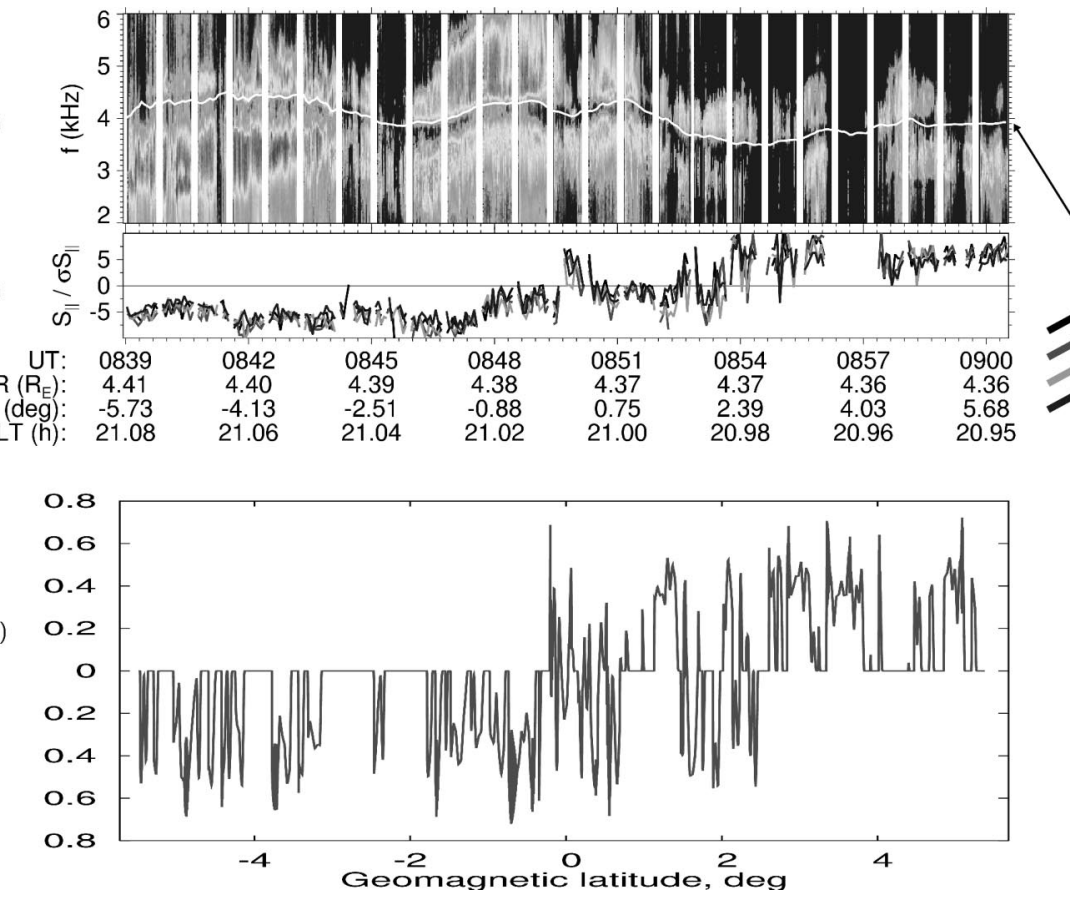

FIG. 3. Upper panel, dynamic spectrum of VLF waves observed by Cluster 1 during its pass through the equatorial region on April 18, 2002 [electric field fluctuations recorded by the WBD instrument (Ref. 20)]. Middle panel, Parallel component of the Poynting vector normalized by its standard deviation from the measurement of the STAFF-SA instruments on board the four Cluster spacecraft (spacecraft indicated by different colors) (Ref. 20). Position is given on the bottom for Cluster 1: $R$, radial distance; MLat, magnetic dipole latitude; MLT, magnetic local time. Lower panel, Poynting flux $\left(A^{+}\right)^{2}-\left(A^{-}\right)^{2}$ (in arbitrary units) resulted from simulations performed using the discrete model (7)-(9). 
for the event studied experimentally in Ref. 20. The dynamic frequency spectrum and the normalized Poynting vector for this event are represented in Figs. 3(a) and 3(b), respectively. ${ }^{24}$ Below, we discuss these estimates and compare the experiment and theory in more detail.

\section{A. Space-time evolution}

Equations (7)-(9) allow us to consider the dynamics of chorus generation. Computer simulations of this dynamics are shown in Fig. 3(c). Here, the center of the source region $(z=0)$ was placed at GMlat $=1^{\circ}$. To calculate the wave amplitude as seen by a satellite moving along the magnetic field line, we generate time series for amplitudes $A^{ \pm}$at the starting points $\mp l_{\mathrm{BWO}} / 2$, respectively, and assume that the amplitudes remain constant while the waves propagate from their starting points. The amplitude is zero upstream of the starting point, i.e., $A^{+}\left(z<-l_{\mathrm{BWO}} / 2\right)=A^{-}\left(z>l_{\mathrm{BWO}} / 2\right)=0$. This scheme yields both amplitudes at any time and at any point $z$, so we can recalculate them onto the satellite trajectory.

For the simulations, the value of $q$ must be chosen. Here, we assume that the step sharpness and amplitude fluctuate randomly, and specify $q$ as a random value distributed uniformly within some range. There are several processes which can lead to a random variation in the parameters of the step. The shortest time scale of such a variation can be related to the reabsorption of a chorus element outside the generation region; this time scale can be of the same order as $T_{0}$. Variations in $q$ can also be related to bounce-oscillations of energetic electrons with time scales $T_{b} \lesssim 1 \mathrm{~s}$. Longer time scales arise due to the finite time of transverse correlation of the electron distribution, which is about the drift time through the BWO source region. According to Ref. 21, the transverse size of the chorus source is about 70-100 km, which yields the drift time about 4-6 s for $60 \mathrm{kev}$ electrons. Source variations can also take place due to the magnetic field fluctuations. Such variations have been reported, e.g., in Ref. 7 and had time scales of several seconds. As it is discussed in the next section, the assumption of a random variation in $q$ is important to explain temporal characteristics of the emissions.

For the result shown in Fig. 3(c), the distribution of $q$ variations was assumed uniform within the range $[0.3,2.3]$ (note that the threshold value of $q=1$ corresponds to the relative height of the step $b=17 \%$, see Table I), and the time scale of $q$ variations was chosen about 4 s. These calculations represent fairly well the chorus dynamics observed by Cluster, as it crossed the equator, moving along the magnetic field line [Fig. 3(b)]. The most important quantitative aspect of this agreement is the good coincidence between the estimated and observed sizes of the chorus source. The latitudinal extent of the source is about $3^{\circ}$, if we use the parameters shown in Table I. If shorter time scales are allowed for the $q$ variation, then the simulated spikes become more frequent, but the overall picture remains the same. Note that the simple discrete model used here does not take into account the amplitude variation along the group ray path and averaging over the detector timescale. Qualitatively, these factors together lead to a smooth variation of the parallel component of the
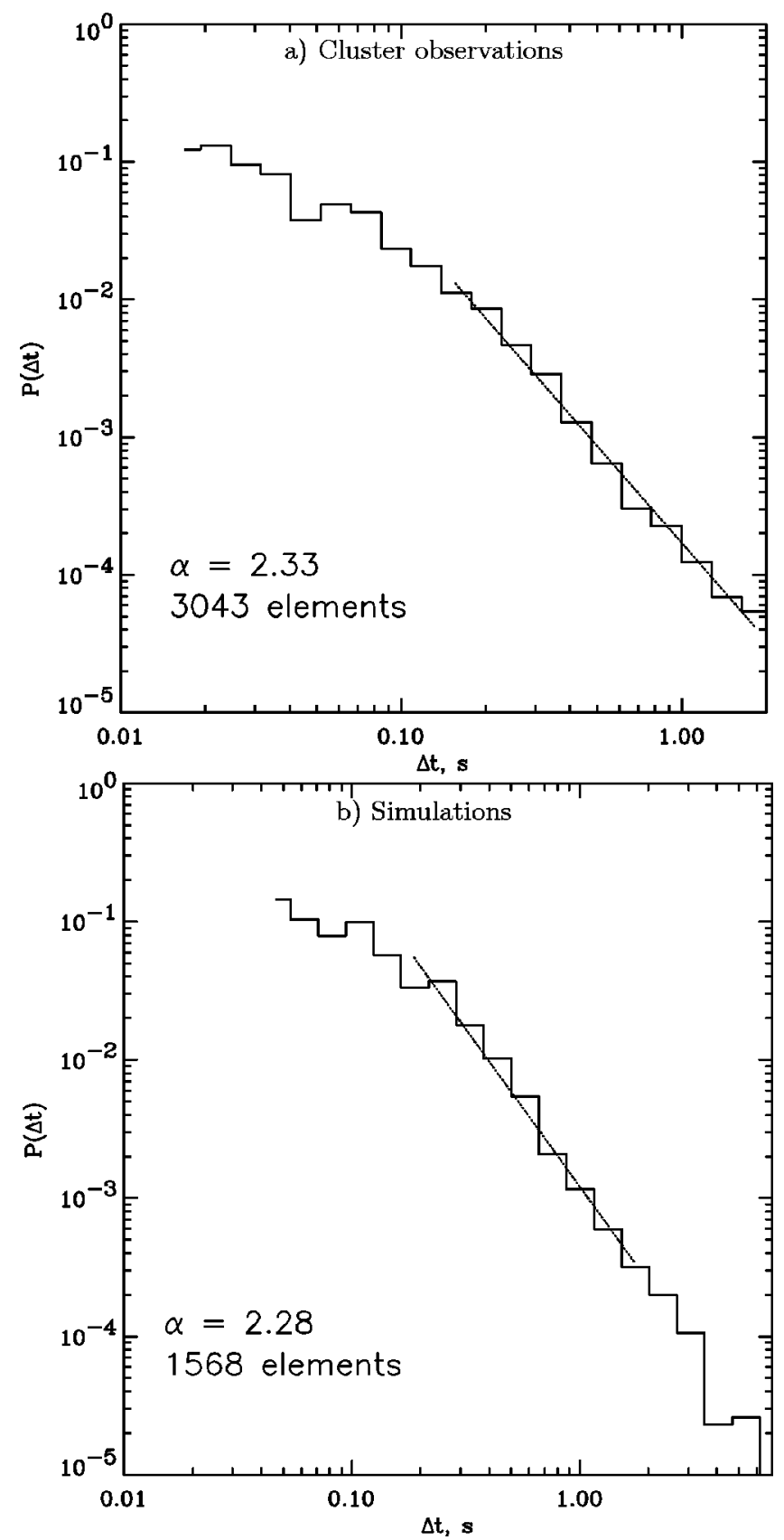

FIG. 4. Distribution of time intervals between chorus elements as seen by Cluster and simulated using the discrete BWO model. On-off intermittency manifests itself by the power-law behavior of the distribution, $P \propto(\Delta t)^{-\alpha}$.

Poynting flux from negative values on the one side of the source region to positive ones, on the other side.

\section{B. On-off intermittency of the VLF chorus}

Statistical analysis of chorus spectrograms obtained from Cluster data (Fig. 3) revealed interesting regularities in the distribution of time intervals between chorus elements. This distribution, shown in Fig. 4(a), could be approximated by the power-law function

$$
P \propto(\Delta t)^{-\alpha},
$$



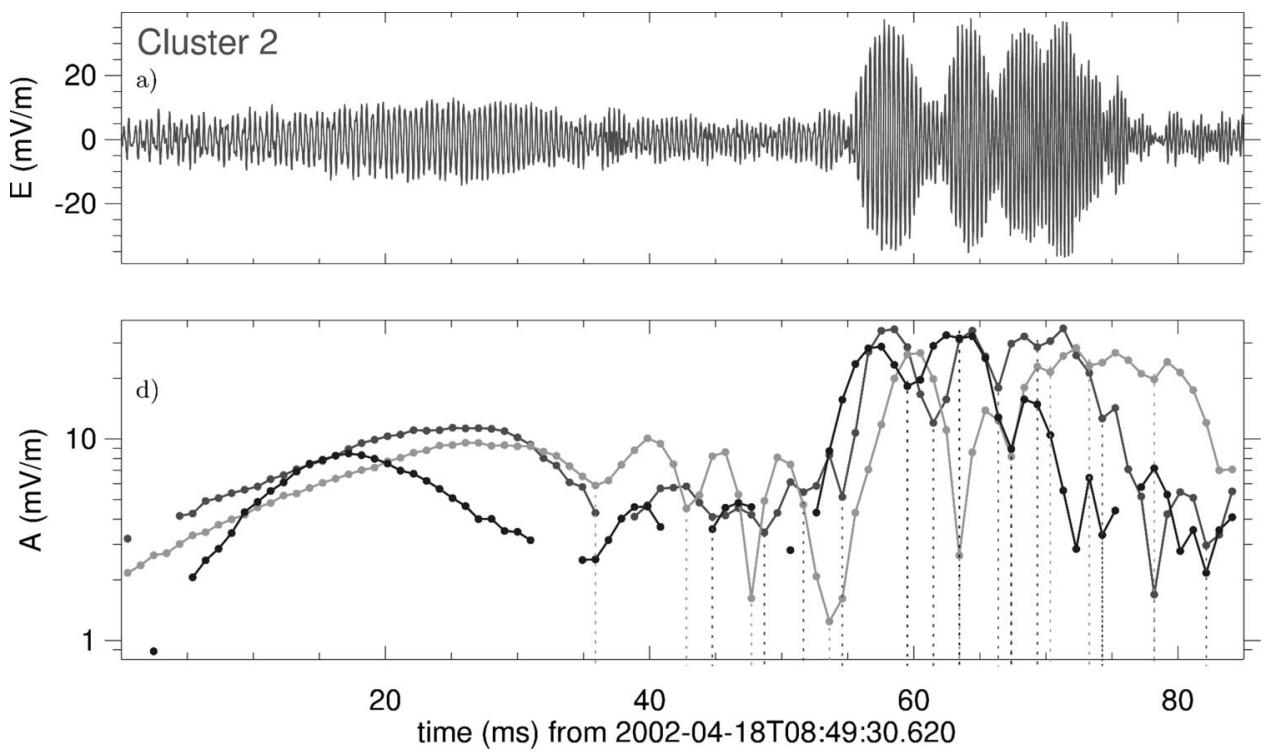

FIG. 5. Panels (a) and (d) from Fig. 6 in Ref. 20, representing fine structure of a chorus element.

where $P$ is the probability density for the time interval $\Delta t$ between chorus elements. Fitting observations to the powerlaw function yields $\alpha \simeq 2.33$.

Such a dependence does not follow from the model (7) to (9) if the coefficients are assumed to be constant. In reality, the parameter $q$ changes in time both regularly and randomly. Calculations show that the power law for $P(\Delta t)$ is obtained if $q$ varies randomly in time. In our calculations, $q$ was changing as a random value distributed uniformly within the interval [0.3-2.3]. In this case, the power-law exponent $\alpha \simeq 2.28$ is close to the experimentally obtained one. Here, we allowed the time scales of the $q$ variation to be as short as the BWO characteristic time $T_{0}$. The possible nature of such variations is discussed in Sec. III A. Actually, the obtained power law for $P(\Delta t)$ corresponds to the well-known "onoff" intermittency regime ${ }^{22}$ with some features specific for the discrete BWO model. More detailed analysis of this problem is presented in Ref. 23. Note that the chosen shortest time scale of the $q$ variation determines the lower limit $(\Delta t)_{\min }$ in the power-law dependence of $P(\Delta t)$, and the power-law exponent is determined mainly by the minimum value of $q$.

\section{Fine structure in wave dynamics}

Figure 5 shows fine structure of the same event as in Fig. 3 with temporal resolution of $1 \mathrm{~ms}^{24}$ One can see some pulses (satellites) varying with characteristic time scale $\tau$ $\sim 10 \mathrm{~ms}$. According to the BWO model, the nonlinear stage of chorus generation is determined by trapping of energetic particles at the step-like feature, which leads to sideband formation at time scale of about $\gamma_{\mathrm{BWO}}^{-1}$. Subsequent bursts are then generated with frequency shift of about $\Omega_{\text {tr }} /(2 \pi)$ one from another.

Therefore, one can expect temporal modulation in VLF wave amplitude, which is confirmed by the plots shown above. Using the parameters specified in Table I, we obtain $\Omega_{\mathrm{tr}} \sim 10^{2}-10^{3} \mathrm{~s}^{-1}$, which corresponds to the temporal scale of fine structure $\tau \sim 6-60 \mathrm{~ms}$.

\section{Relation between chorus and hiss emissions}

Figure 6 shows an example of good correlation between chorus and hiss emissions, where groups of chorus elements $(f=2-3 \mathrm{kHz})$ are observed simultaneously with separate hiss bursts at a lower frequency. ${ }^{24}$ This corresponds qualita-

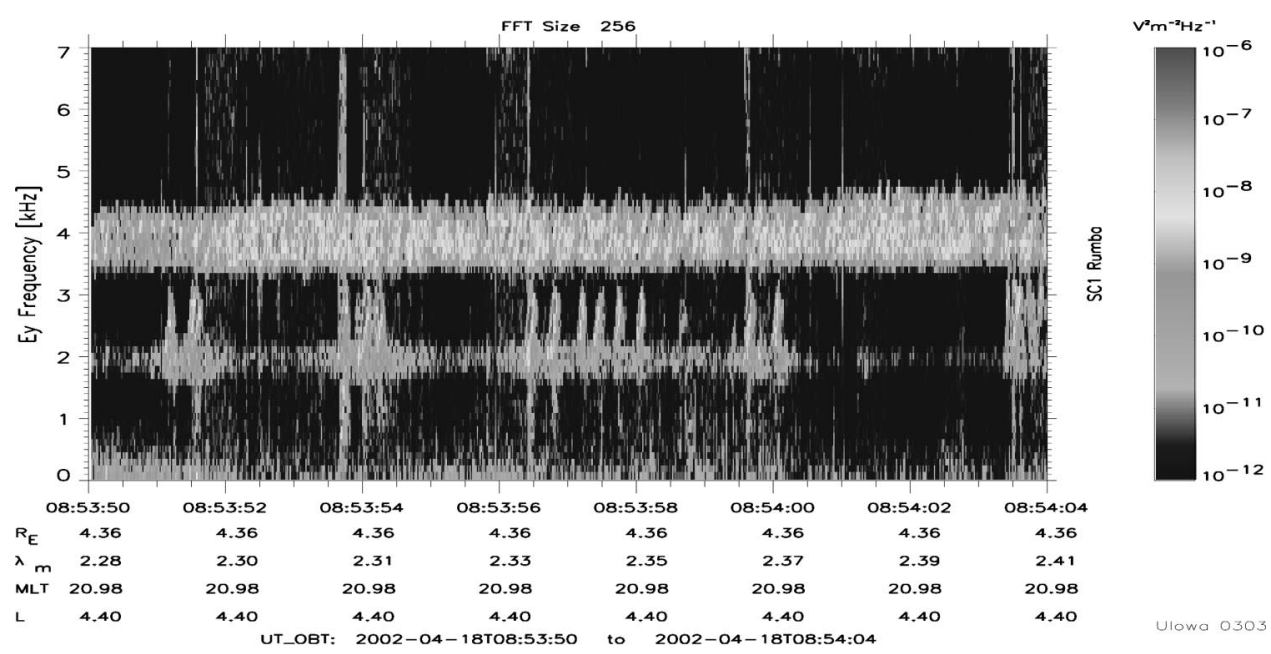

FIG. 6. An example of good correlation between chorus and hiss emissions in Cluster data. 
tively to the BWO model. ${ }^{12}$ However, it is noteworthy that such close correlation between chorus and hiss is not always present in Cluster observations. We think that sharp gradients in the phase space, which are needed for switching on the absolute instability, can appear at the developed phase of the BWO generation due to the reabsorbtion of a chorus element, as it propagates along the magnetic flux tube from its central cross section and interacts with new incoming electrons. It would be important to find experimental evidences of such effects.

\section{CONCLUSIONS}

The BWO generation model is a good candidate to explain quantitatively chorus emissions at frequencies below $f_{H} / 2$, observed by Cluster satellites. Within the framework of this model, it is possible to explain the properties of the chorus source, such as its location and the direction of the energy flux, the chorus growth rate, the frequency drift, and the amplitude.

At the same time, some principal questions remain unclear. First, it concerns relation between chorus and hiss emissions and formation of a step-like distortion at the electron distribution function.

The second question is which processes determine the correlation of chorus emissions across the geomagnetic field, in particular, whether there are whistler mode ducts in the generation region.

It is important to study the chorus emissions at frequencies $f>f_{H} / 2$, in particular, to check if there is a relation between the chorus above and below $f_{H} / 2$.

In the field of theory, computer simulations of the step formation using nonquasilinear approach are needed, such as hybrid simulations. ${ }^{18}$ In this approach, chorus generation should be a part of the common process of the cyclotron instability development.

\section{ACKNOWLEDGMENTS}

A.D. and V.T. acknowledge support from the Russian Foundation for Basic Research (Grant No. 02-02-17109) and from the Program for Basic Research of the Russian Academy of Sciences. Part of this work was done during the visit of V.T., A.D., E.T., B.K., and O.S. to Sodankylä Geophysical Observatory, supported by the EU LAPBIAT program, and during the visit of E.T. and V.T. to Bern, Switzerland, supported by International Space Science Institute. O.S. and
M.P. acknowledge discussions of the STAFF data with N. Cornilleau-Wehrlin, C. Harvey, M. Maksimovic, and Y. de Conchy. We thank P. Décréau and P. Canu for estimating the plasma density from the data of the Whisper experiment, and the access to the spin-resolution data of the FGM magnetic field experiment (PI A. Balogh) used for reference. The WBD research was supported by the NASA Goddard Space Flight Center under Grant No. NAG5-9974. O.S. acknowledges additional support from Grants Nos. ME 650 and GACR 202/03/0832.

${ }^{1}$ R. A. Helliwell, Rev. Geophys. 7, 281 (1969).

${ }^{2}$ S. S. Sazhin and M. Hayakawa, Planet. Space Sci. 40, 681 (1992).

${ }^{3}$ R. B. Horne and R. M. Thorne, Geophys. Res. Lett. 30, 1527 (2003).

${ }^{4}$ J. L. Roeder, J. R. Benbrook, E. A. Bering, and W. R. Sheldon, J. Geophys. Res. 90, 10975 (1985).

${ }^{5}$ N. P. Meredith, M. Cain, R. B. Horne, R. M. Thorne, and R. R. Anderson, J. Geophys. Res. 108, 1248 (2003).

${ }^{6}$ M. J. LeDocq, D. A. Gurnett, and G. B. Hospodarsky, Geophys. Res. Lett. 25, 4063 (1998).

${ }^{7}$ M. Parrot, O. Santolik, N. Cornilleau-Wehrlin, M. Maksimovic, and C. Harvey, Ann. Geophys. 21, 473 (2003).

${ }^{8}$ R. A. Helliwell, Whistlers and Related Ionospheric Phenomena (Stanford University Press, Palo Alto, CA, 1965).

${ }^{9}$ R. A. Helliwell, J. Geophys. Res. 72, 4773 (1967).

${ }^{10}$ Y. Omura, D. Nunn, H. Matsumoto, and M. J. Rycroft, J. Atmos. Terr. Phys. 53, 351 (1991).

${ }^{11}$ D. Nunn, Y. Omura, H. Matsumoto, I. Nagano, and S. Yagitani, J. Geophys. Res. 102, 27083 (1997).

${ }^{12}$ V. Y. Trakhtengerts, Ann. Geophys. 17, 95 (1999).

${ }^{13}$ V. Y. Trakhtengerts, J. Geophys. Res. 100, 17205 (1995).

${ }^{14}$ N. S. Ginzburg and S. P. Kuznetsov, Relativistic HF Electronics (Inst. of Appl. Phys., Gorky, USSR, 1981), pp. 101-144, in Russian.

${ }^{15}$ V. Y. Trakhtengerts, M. J. Rycroft, and A. G. Demekhov, J. Geophys. Res. 101, 13293 (1996).

${ }^{16}$ A. G. Demekhov, D. Nunn, and V. Y. Trakhtengerts, Phys. Plasmas 10, 4472 (2003).

${ }^{17}$ D. Nunn, Comput. Phys. Commun. 60, 1 (1990).

${ }^{18}$ D. Nunn, A. G. Demekhov, V. Y. Trakhtengerts, and M. J. Rycroft, Ann. Geophys. 21, 481 (2003).

${ }^{19}$ D. Nunn, Planet. Space Sci. 22, 349 (1974).

${ }^{20}$ O. Santolik, D. A. Gurnett, J. S. Pickett, M. Parrot, and N. CornilleauWehrlin, J. Geophys. Res. 108, 1278 (2003).

${ }^{21}$ O. Santolík and D. A. Gurnett, Geophys. Res. Lett. 30, 1031 (2003).

${ }^{22}$ J. F. Heagy, N. Platt, and S. M. Hammel, Phys. Rev. E 49, 1140 (1994).

${ }^{23}$ B. V. Kozelov, E. E. Titova, A. A. Lubchich, V. Y. Trakhtengerts, and J. Manninen, Geomagn. Aeron. 43, 593 (2003).

${ }^{24}$ See EPAPS Document No. E-PHPAEN-11-032404 for color versions of Figs. 3, 5, and 6 of this paper. A direct link to this document may be found in the online article's HTML reference section. The document may also be reached via the EPAPS homepage (http://www.aip.org/pubservs/ epaps.html) or from ftp.aip.org in the directory /epaps/. See the EPAPS homepage for more information. 